n'est, en somme, qu'une polymérisation et une hydratation progressive des micelles » (p. 224-225.)

Quels sont les rapports entre la gélification du lait par les facteurs physiques ou chimiques et celle par la présure?

Ici commence un no man's land expérimental. Nous ignorons le mécanisme des diverses actions catalytiques. Signalons, toutefois, deux analogies entre ces deux Imodes de gélification : on retrouve dans les deux cas l'influence du facteur temps et celui de la température, ainsi que l'apparition d'un grossissement micellaire dans l'action de la présure sur du lait, dégraissé au maximum par la centrifugation.

C'est de telle façon que l'on peut comprendre, à l'état actuel de nos connaissances, le mécanisme de la gélification du lait. Nous apporterons à la fin de ce travail d'autres arguments per analogiam en faveur de cette conception.

\title{
REVUE
}

\section{L'INDUSTRIE LAITIÈRE DANS LE MONDE}

par

G. GÉNIN

Ingénieur E. P. C.

\section{ÉTATS-UNIS}

\section{La valeur alimentaire du sérum}

Le Dr L. K. Rigas a publié récemment une étude sur l'utilisation du sérum dans laquelle il signale que la protéine du sérum, connue sous le nom de lactalbumen, présente une valeur biologique extraordinairement élevée. Du point de vue nutritif, sa valeur biologique est de $96,4 \%$, par rapport à $85,5 \%$ pour la caséine.

Or on sait qu'actuellement le monde entier manque de protéines de haute valeur nutritive, et s'il était possible d'utiliser la totalité de lactalbumen contenu dans le sérum, il deviendrait facile de fournir aux hommes une quantité considérable d'un produit naturel de haute valeur.

Lorsque le sérum est manipulé avec autant de soin que le lait frais, puis pasteurisé et desséché, il donne un produit de haute qualité et de goût excellent. Jusqu'à présent, cette substance a surtout été utilisée pour la nourriture du bétail et pour l'alimentation des volailles. On l'a cependant déjà employé également pour la nourriture de l'homme, par exemple comme constituant de certains fromages, ainsi que dans la fabrication de gâteaux et de la pâtisserie. 
Dans l'industrie, le sérum a été également employé dans la fabrication de l'acide lactique et de ses dérivés, ainsi que dans la production d'alcool et du vinaigre.

Si dans l'industrie, le sérum est capable de trouver encore d'autres applications, le Dr RIGGs estime que c'est surtout comme aliment qu'on devrait envisager ses emplois et c'est à l'industrie laitière de s'efforcer de trouver des débouchés dans cette voie.

\section{Le développement de l'industrie laitière}

M. H. L. Cook qui appartient au Bureau de l'Economie agricole des Etats-Unis a publié récemment une étude sur la contribution de l'industrie à l'utilisation totale des substances nutritives du lait.

Le tableau ci-dessous indique pour chacun des produits nutritifs contenus dans le lait qui, rappelons-le, a été produit en 1944 en quantité atteignant près de 60 milliards de kilogrammes aux EtatsUnis, le pourcentage de ces substances utilisées dans chacune des formes essentielles de consommation du lait.

\begin{tabular}{|c|c|c|}
\hline$\rightarrow$ & $\begin{array}{l}\text { Produits } \\
\text { nutritifs } \\
\text { totaux }\end{array}$ & $\begin{array}{c}\text { Produits nutritif's } \\
\text { non dégraissés } \\
\%\end{array}$ \\
\hline Lait fluide .... . & $\ldots \ldots \ldots \ldots \ldots$ & 66,02 \\
\hline Lait coneentré. & $\ldots \ldots \ldots \ldots \ldots \ldots$ & 11,08 \\
\hline Lait en poudre & $\ldots \ldots$ & 12,51 \\
\hline Fromage ..... & 5,74 & 5,16 \\
\hline Crème glacée. & $\ldots \ldots \ldots \ldots \ldots \ldots$ & 3,55 \\
\hline
\end{tabular}

En 1944, le lait produit contenait environ 7 milliards de kilogrammes de produits nutritifs. Sur ce total, environ 5 milliards 5 de kilogrammes ont été utilisés pour l'alimentation humaine.

Le calcium est un des plus importants produits nutritifs contenus dans le lait. C'est également le lait et les produits laitiers qui fournissent à l'homme la grande partie de la vitamine A dont il a besoin, des proportions importantes de riboflavine, ainsi que des petites quantités de thiamine, de vitamine $\mathrm{C}$ et de niacine.

\section{La lutte contre la corrosion des chaudières}

On sait que le gaz carbonique dissous dans les eaux de condensation est une des causes essentielles des phénomènes de corrosion. que l'on constate dans les chaudières, phénomène de corrosion dû à l'action de la vapeur ou des eaux de condensation contenant cet acide carbonique.

On peut réduire ce phénomène par l'addition aux eaux de morpholine qui est un agent alcalin organique, dont la présence permet de réduire l'acidité due à la présence de gaz carbonique libre dans les eaux d'alimentation ou à la présence de gaz carbonique résultant 
de la décomposition des carbonates solubles. Ce produit s'évapore avec l'eau, de telle sorte qu'il est possible de maintenir dans la vapeur produite par une chaudière une concentration déterminée en morpholine et de réduire ainsi l'action corrosive de la vapeur;

\section{Un nouveau mode d'emballage du camembert}

Pour la première fois, on a utilisé pour l'emballage du camembert des boîtes en matière plastique en forme de demi-lune, complètement transparentes et qui renferment un certain nombre de parts de camembert, elles-mêmes enveloppées dans un papier métallique.

Ce qui fait l'intérêt de ce nouveau mode d'emballage, c'est qu'il peut être utilisé après emploi par la ménagère pour un grand nombre d'applications.

Par ailleurs, l'expérience a montré que le fromage quiest un produit particulièrement délicat mûrit parfaitement dans cet emballage et qu'il ne perd par conséquent aucune de ses qualités. A la suite de premiers essais d'emballage de cette forme, l'idée a paru tellement séduisante à la clientèle que la décision a été prise de le généraliser.

\section{Un nouvel essai pour le contrôle de la pasteurisation}

Le Bureau de l'Industrie laitière du Ministère de l'Agriculture vient de publier un nouveau mode d'essai à la phosphatase, en vue de déterminer si le lait utilisé pour la fabrication du fromage Cheddar a été pasteurisé ou non. On a constaté en outre que ce même essai permet également de déterminer si d'autres produits laitiers, tels que lait, crème, fromage à pâte molle ou à pâte dure, beurre, petit-lait, mélange pour crème glacée, sérum, fromage de chèvre etc., ont été également pasteurisés.

Le nouveau procédé connu sous le nom d'essais Sanders et Sager est une modification de l'ancien procédé Scharer. Il repose sur le fait que tout lait normal cru renferme un enzyme qui a la propriété d'être détruit à une température supérieure de quelques degrés à celle qui est nécessaire pour détruire les plus résistants de tous les organismes pathogènes existant dans le lait.

Par ce procédé, il est possible de déceler une diminution de l'ordre d'un degré C. de la température de pasteurisation. Cet essai est en outre suffisamment sensible pour lui permettre de déceler la présence d'une partie de lait eru dans 2.000 parties de lait convenablement pasteurisé.

\section{La fabrication continue du beurre}

Deux procédés pour la fabrication continue du beurre ont été récemment mis au point. Dans la méthode Cherry-Burrell, on procède à la conservation de la crème dans un appareil centrifuge, de 
façon à élever sa teneur en graisse à 86-90\%. Le produit subit ensuite une pasteurisation très rapide à environ $90^{\circ}$ pendant quelques secondes, puis il est refroidi à environ $45^{\circ}$. La crème est alors standardisée par addition d'un sel, neutralisée s'il y a lieu, puis additionnée si on le désire d'un colorant et d'un démarreur. Finalement, le mélange est dirigé par une pompe dans un appareil réfrigérant ressemblant à un appareil pour la fabrication en continu de la crème glacée. Dans ces conditions, la graisse cristallise, elle est alors travaillée dans un "texturateur " puis emballée. Le beurre ainsi produit se caractérise par une structure très compacte.

- Dans le procédé "Creamery package », la crème est neutralisée, pasteurisée, puis chauffée à environ $75^{\circ}$, concentrée de façon à élever sa teneur en graisse à $75-80 \%$ et traitée dans un homogénéiseur. On sépare alors la graisse du sérum par décantation, et la graisse liquéfiée s'écoule entre les deux cylindres d'une pompe à piston Triplex. Une solution saline s'écoule dans le troisième cylindre de la pompe et se mélange avec la graisse. A la sortie de l'appareil, le mélange qui est à la température de $60^{\circ}$ environ, passe dans un réfrigérant analogue à un appareil de fabrication de crème glacée où la graisse se solidifie. Celle-ci est alors travaillée pour obtenir la structure convenable, puis emballée automatiquement.

\section{AMÉRIQUE CENTRALE}

\section{La production laitière}

Les pays de l'Amérique centrale se caractérisent par une production très réduite de lait et par conséquent par un manque de produits laitiers nécessaires à l'alimentation humaine. La production du lait sur place est toujours très faible et les importations de produits laitiers sont en général insuffisantes pour répondre aux demandes. Cependant, depuis quelques années, on constate la création de quelques nouveaux établissements industriels qui manquent d'ailleurs de matériel permettant d'obtenir des produits répondant à des standards élevés de qualité.

Cependant, à Cuba, les pluies abondantes de l'annés 1947 ont permis de maintenir les pâturages en bon état et la production de lait au cours de l'année est la plus forte que l'on ait enregistré depuis 1943. Elle est estimée à environ 500 millions de kilogrammes, comparée à des chiffres qui ne dépassaient guère 400 millions de kilogrammes en 1945 et 1946 . Le nombre du bétail a été en diminuant depuis plusieurs années ; on pense néanmoins que certaines plantations de betteraves à sucre seront transformées en pâturage. Cette tendance se généralisant si, comme on le pense, la demande de produits laitiers va en eroissant. 
L'industrie laitière au Mexique a subi une éclipse du fait d'épidémies qui ont frappé les troupeaux de vaches laitières vers la fin de 1946. Ces épidémies se sont en particulier étendues au voisinage de Mexico, ce qui a obligé les proprétaires de bétail à abattre leurs animaux. Il faudra donc procéder à une reconstitution des troupeaux en important des Etats-Unis et du Canada de nouveaux animaux reproducteurs. En attendant, les importations de produits laitiers des Etats-Unis sont en augmentation.

Le département américain de l'agriculture, en collaboration avec les gouvernements de l'Amérique centrale, étudie actuellement l'emploi de pulpe de café pour la nourriture du bétail, en vue du remplacement par ce produit du maïs ou d'autres céréales utilisées jusqu'alors. Le manque de céréales a donné une grande impulsion à ces recherches et il n'est pas déraisonnable de penser que la pulpe de café deviendra à l'avenir un important produit pour l'alimentation du bétail dans tous les pays qui sont de grands producteurs de café ou dans les pays avoisinants.

\section{HOLLANDE}

\section{Une nouvelle revue laitière}

Dans le courant de l'année 1947, une nouvelle revue laitière a fait son apparition aux Pays-Bas. Jusqu'à présent, eette Revue ne paraît que tous les trois mois, sous le titre de « Journal du Lait et de 1a Laiterie des Pays-Bas ". Les articles d'intérêt général sont rédigés en anglais ou en langue europóenne et les artieles d'intérêt locàl seuls sont rédigés en hollandais. Ce journal est édité par l'Association pour l'avancement de la science du lait, en collaboration avec le Ministère hollandais de l'agriculture, des pêcheries et des produits alimentaires.

Le montant de la souseription est actuellement de 8 florins et l'adresse de l'éditeur est :

D. B. Centen's Ultgevers Mij. N. V.

Sarphatikade 12, Amsterdam (Holland).

Parmi les articles publiés dans les premiers numéros, citons les études sur l'instabilité du lait, due à une activité accrue des ions calcium, sur la valeur nutritive du lait, sur l'appréciation de la qualité bactériologique du lait au moyen de l'essai à la rézazurine et sur l'état de la caséine dans le lait.

\section{Un nouveau produit sucrant}

Pendant la guerre, on a commencé aux Pays-Bas la fabrication d'un nouveau produit sucrant, destiné à remplacer le sucre dans certaines de ses applications et dont le pouvoir sucrant est 4.000 
fois supérieur à celui du sucre. Ce produit qui est le 1-N propoxy-2amino-4 nitrobenzène, se présente sous la forme d'un produit pulvérulent, cristallisé de coloration orange.

Sa solubilité dans l'eau est faible, mais il reste stable à la température de l'eau bouillante. Pratiquement, on l'emploie en mélange avec le lactose et sous cette forme il constitue un produit dont le pouvoir sucrant est 500 fois supérieur à celui du sucre.

\section{SUISSE}

\section{La fabrication du fromage}

Le tableau ci-dessous indique les variations de la production et de la livraison de fromages au cours des semestres d'hiver de ces dernières années :

\begin{tabular}{|c|c|c|}
\hline Année & $\begin{array}{c}\text { Production en } \\
\text { quintaux }\end{array}$ & $\begin{array}{l}\text { Livraison en } \\
\text { quintaux }\end{array}$ \\
\hline $1939-40$ & 87.600 & 79.311 \\
\hline $1940-41$ & 78.615 & 71.393 \\
\hline 1941.42 & 97.289 & 85.875 \\
\hline 1942.43 & 112.374 & 96.806 \\
\hline $1943-44$ & 58.800 & 46.099 \\
\hline $1944-45$ & 78.702 & 66.318 \\
\hline $1945-46$ & 78.064 & 65.793 \\
\hline $1946-47$ & 70.532 & 59.662 \\
\hline
\end{tabular}

La modification du programme général de fabrication découlant pendant la guerre de l'intensification de la fabrication du beurre, a déterminé une fabrication plus marquée des fromages maigres. Cette fabrication particulière a augmenté d'année en année, mais la qualité des fromages maigres et des fromages gras a fait, comme d'habitude, l'objet d'un contrôle très sévère des organismes syndicaux.

\section{La production et la mise en valeur du lait}

C'est la production de fourrage vert et sec qui constitue le fondement de l'alimentation des animaux suisses. Aussi la qualité des fourrages et leur teneur en valeur nutritive sont autant d'éléments qui exercent leur influence sur la production laitière. La disette fourragère a sensiblement retardé la reconstitution des troupeaux qui ont diminué pendant la guerre, et en outre on constate un abandon de l'exploitation du bétail.

En une année se terminant au milieu de 1947, le troupeau a diminué de $1,5 \%$ ce qui représente une diminution par rapport à 1939 de $15,2 \%$. Certes, cette diminution porte surtout sur les bœufs, alors que le nombre de vaches a très légèrement augmenté, mais il s'agit là de bêtes très jeunes. 
La production laitière totale est estimée en 1946 à 21.600 .000 quintaux, supérieure de $1,9 \%$ à celle de 1945 , mais inférieure de $19,8 \%$ à celle de 1939. L'utilisation du lait produit s'est répartie comme suit :

\begin{tabular}{|c|c|c|c|c|c|}
\hline & & & Moyenne en & quintaux & \\
\hline Utilisation du lait & 1939 & .1943 & 1944 & 1945 & 1946 \\
\hline Elevage...$\ldots \ldots$. & 4.04 & 0.000 & 3.200 .000 & 3.200 .000 & 3.200 .00 \\
\hline Consommation des & & & & & \\
\hline producteurs .... & 3.78 & 0.000 & 3.700 .000 & 3.700 .000 & 3.700 .00 \\
\hline $\begin{array}{c}\text { Vendu à la consom- } \\
\text { mation } . . . . . .\end{array}$ & 6.63 & .000 & 6.087 .000 & 6.085 .000 & 6.280 .00 \\
\hline $\begin{array}{c}\text { Transformé en pro- } \\
\text { duits laitiers .... }\end{array}$ & 10.06 & 8.000 & 8.163 .000 & 8.215 .000 & 8.420 .00 \\
\hline $\begin{array}{l}\text { Quant aux pro } \\
\text { partissent comm }\end{array}$ & is & tiers & rio & par & lait, ils s \\
\hline & & & Moyenne e & en quintaux & \\
\hline & & 1943 & 1944 & 1945 & 1946 \\
\hline Fromage & & 438.000 & 423.000 & 432.000 & 421.000 \\
\hline Beurre. & $\cdots$ & 190.000 & 187.000 & 182.000 & 196.000 \\
\hline Autres produits. & $\ldots$ & 108.000 & 127.000 & 110.000 & 127.000 \\
\hline
\end{tabular}

\section{AUTRICHE}

\section{La situation de la production laitière}

En 1938, l'Autriche couvrait non seulement ses besoins en lait et produits laitiers, mais elle exportait également certains de ces produits, comme par exemple les spécialités fabriquées en Autriche: l'Emmenthal, l'Edam autrichien, les fromages à pâte molle et le yoghourt, ainsi que du beurre. Après l'occupation, l'industrie laitière autrichienne était pratiquement ruinée et tout ce que l'occupation avait laissé intact fut détruit par les troupes allemandes au moment de leur départ.

En 1937, la production laitière mensuelle de l'Autriche était de 196.000 tonnes; pendant la guerre, elle ne fut plus que de 160.000 tonnes par mois, en 1946, elle était tombée à 105.000 tonnes par mois et en 1947, elle n'a guère dépassé ce chiffre.

Il en résulte que les besoins actuels minima de la population ne peuvent être assurés et seuls les très jeunes enfants reçoivent de très faibles rations journalières. C'est surtout le manque de denrées fourragères, par suite de la sècheresse qui a exercé une néfaste influence sur la production, et en 1948 , il est peu probable que les livraisons augmentent sensiblement, malgré les mesures diverses envisagées actuellement : augmentation du prix du lait, octroi de primes en nature aux fermiers telles que livraison d'engrais chimiques, de fourrage, de machines agricoles, d'habits et de chaussures. 


\section{AUSTRALIE}

\section{Beurre pour les tropiques}

Développé au cours de la guerre, un procédé nouveau pour la fabrication du beurre déshydraté a été mis au point en Australie. Il permet de préparer un produit qui, fondant à $40^{\circ} \mathrm{C}$., peut être conservé pendant de longues périodes sans qu'il soit nécessaire de le réfrigérer.

Ce produit est constitué essentiellement de graisse de beurre sèche, à laquelle on ajoute $3 \%$ d'huile d'arachide hydrogénée, $4 \%$ de poudre de lait écrémé, $2 \%$ de sel finement broyé, et une petite quantité de diacétyle destiné à améliorer le goût. A température ordinaire, ce beurre s'étale sur le pain aussi facilement que le beurre natúrel.

La fabrication s'effectue essentiellement dans un appareil exécuté entièrement en acier inoxydable. Le $p \mathbf{H}$ du sérum du lait est réglé à environ 10 dans des cuves de neutralisation par addition de soude caustique, ou de carbonate de sodium, le produit est ensuite centrifugé et la crème est desséchée par atomisation, en opérant dans le vide et à la température maximum de $70^{\circ}$, de façon à abaisser la teneur en humidité du produit obtenu à moins de $0,2 \%$. La graisse ainsi desséchée passe dans un clarificateur pour éliminer les dernières traces de sel et de caillebotte, puis dans un second déshydrateur, fonctionnant à une température plus élevée, l'humidité est abaissée à $0,05 \%$, en même temps que le produit est partiellement désaéré. On le refroidit ensuite rapidement et il est introduit dans des boîtes hermétiques.

\section{Commerce d'exportation des produits laitiers}

Dans la liste des pays les plus importants en tant qu'exportateurs et producteurs de lait et de produits laitiers, l'Australie et la Nouvelle-Zélande occupent une place importante, mais leur production reste néanmoins inférieure à celle d'avant-guerre et bien inférieure à celle de 1942 qui avait constitué une année record.

Si l'état des pâturages est plus favorable que celui des années précédentes, le nombre des vaches laitières est en réduction et les producteurs rencontrent beaucoup de difficulté à attirer la maind'œuvre et à se procurer les engrais nécessaires au bon entretien des prairies. En Nouvelle-Zélande, la situation est analogue, et le manque d'engrais se fait également sentir. Les prix accordés aux fermiers pour le lait et les produits laitiers se sont trouvés influencés par l'accord à long terme signé entre l'Australie et l'Angleterre. 